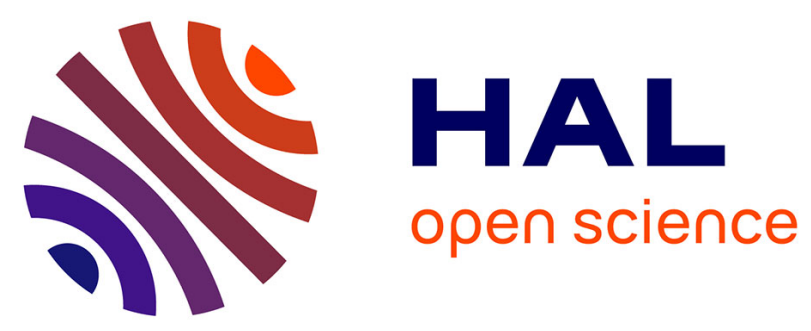

\title{
Expression of a truncated tobacco NtCBP4 channel in transgenic plants and disruption of the homologous Arabidopsis CNGC1 gene confer $\mathrm{Pb} 2+$ tolerance
}

\author{
Ramanjulu Sunkar, Boaz Kaplan, Nicolas N. Bouche, Tzahi Arazi, Dvora
}

Dolev, Ina N. Talke, Frans J.M. Maathuis, Dale Sanders, David D. Bouchez, Hillel Fromm

\section{To cite this version:}

Ramanjulu Sunkar, Boaz Kaplan, Nicolas N. Bouche, Tzahi Arazi, Dvora Dolev, et al.. Expression of a truncated tobacco NtCBP4 channel in transgenic plants and disruption of the homologous Arabidopsis CNGC1 gene confer Pb2+ tolerance. Plant Journal, 2000, 24 (4), pp.533-542. 10.1046/j.1365313x.2000.00901.x . hal-02698867

\section{HAL Id: hal-02698867 https://hal.inrae.fr/hal-02698867}

Submitted on 1 Jun 2020

HAL is a multi-disciplinary open access archive for the deposit and dissemination of scientific research documents, whether they are published or not. The documents may come from teaching and research institutions in France or abroad, or from public or private research centers.
L'archive ouverte pluridisciplinaire HAL, est destinée au dépôt et à la diffusion de documents scientifiques de niveau recherche, publiés ou non, émanant des établissements d'enseignement et de recherche français ou étrangers, des laboratoires publics ou privés. 


\title{
Expression of a truncated tobacco NtCBP4 channel in transgenic plants and disruption of the homologous Arabidopsis CNGC1 gene confer $\mathrm{Pb}^{2+}$ tolerance
}

\author{
Ramanjulu Sunkar ${ }^{1, \dagger}$, Boaz Kaplan ${ }^{1, \dagger}$, Nicolas Bouché ${ }^{3, \dagger}$, Tzahi Arazi ${ }^{1}$, Dvora Dolev ${ }^{1}$, Ina N. Talke ${ }^{4}$, Frans J.M. Maathuis ${ }^{4}$, \\ Dale Sanders ${ }^{4}$, David Bouchez ${ }^{3}$ and Hillel Fromm ${ }^{1,2, *}$ \\ ${ }^{1}$ Department of Plant Sciences, The Weizmann Institute of Science, Rehovot 76100, Israel, \\ ${ }^{2}$ Centre for Plant Sciences, Leeds Institute for Biotechnology and Agriculture (LIBA), School of Biology, University of \\ Leeds, Leeds LS2 9JT, UK, \\ ${ }^{3}$ INRA, Laboratoire de Biologie Cellulaire, Route de Saint Cyr, 78026 Versailles, France, and \\ ${ }^{4}$ The Plant Laboratory, Department of Biology, University of York, York YO1 5YW, UK
}

Received 21 July 2000; revised 8 September 2000; accepted 15 September 2000.

*For correspondence (Centre for Plant Sciences, LIBA, UK; fax +44 113233 3144; e-mail h.fromm@leeds.ac.uk).

${ }^{\dagger}$ These authors contributed equally to this work.

\begin{abstract}
Summary
Recently we reported on a plasma membrane tobacco protein (designated NtCBP4) that binds calmodulin. When overexpressed in transgenic plants, NtCBP4 confers $\mathrm{Pb}^{2+}$ hypersensitivity associated with enhanced accumulation of this toxic metal. To further investigate possible modulation of $\mathrm{Pb}^{2+}$ tolerance in plants, we prepared transgenic plants that express a truncated version of this protein (designated NtCBP4 $\triangle \mathrm{C}$ ) from which its $\mathrm{C}$-terminal, with the calmodulin-binding domain and part of the putative cyclic nucleotide-binding domain, was removed. In contrast to the phenotype of transgenic plants expressing the full-length gene, transgenic plants expressing the truncated gene showed improved tolerance to $\mathrm{Pb}^{2+}$, in addition to attenuated accumulation of this metal. Furthermore, disruption by T-DNA insertion mutagenesis of the Arabidopsis CNGC1 gene, which encodes a homologous protein, also conferred $\mathrm{Pb}^{2+}$ tolerance. We suggest that NtCBP4 and AtCNGC1 are components of a transport pathway responsible for $\mathrm{Pb}^{2+}$ entry into plant cells.
\end{abstract}

Keywords: calcium, cyclic nucleotides, heavy metals, ion channel, knockout mutant, T-DNA.

\section{Introduction}

The characterization of plant genes that code for proteins involved in the transport of heavy metals should be useful in developing appropriate biotechnologies to clean the environment (e.g. phytoremediation; Raskin, 1996; Terry and Banuelos, 1999), as well as for engineering plant tolerance to toxic metals. Because certain metals such as lead $\left(\mathrm{Pb}^{2+}\right)$ are non-essential and toxic to plants, plants are unlikely to have transporters specific for these metals. Instead, the permeability of the plasma membrane to these metals is attributed to the inherently limited selectivity of certain ion transporters.

A family of channel proteins from barley (HvCBT1, Schuurink et al., 1998); Arabidopsis (AtCNGC1-AtCNGC6, Köhler et al., 1999; Leng et al., 1999); and tobacco (NtCBP4, Arazi etal., 1999) was recently reported. In Arabidopsis, at least 19 CNGC (cyclic nucleotide-gated ion channel)-related genes are distributed among the five chromosomes ( $\mathrm{N}$. Bouché, unpublished results), with some of them clustered (for example, genes At2g46430, At2g46440 and At2g46450 on chromosome 2). Structurally, all these proteins contain six putative transmembrane domains, a presumed pore region located between the fifth and sixth transmembrane domains, and a putative cyclic nucleotide monophosphatebinding domain coinciding with a calmodulin-binding site located at the C-terminus (Arazi etal., 2000; Köhler and Neuhaus, 2000). These proteins are similar in overall structure to mammalian cyclic nucleotide-gated non-selective cation channels, implicated in $\mathrm{Ca}^{2+}$ signal transduction.

Various approaches have been used to investigate the function of this plant protein family. Köhler et al. (1999) 
reported that two members of the Arabidopsis gene family (AtCNGC1 and AtCNGC2) could partially complement a $\mathrm{K}^{+}$uptake-deficient yeast mutant. Leng etal. (1999), using heterologous expression systems, suggested that AtCNGC2 is a cyclic nucleotide-stimulated cation channel permeable to $\mathrm{Ca}^{2+}$ and $\mathrm{K}^{+}$. Interestingly, $\mathrm{Pb}^{2+}$ is a highly toxic metal that can bind to $\mathrm{Ca}^{2+}$-binding sites in regulatory proteins such as calmodulin (Ouyang and Vogel, 1998). The similarity in protein-binding sites for $\mathrm{Pb}^{2+}$ and $\mathrm{Ca}^{2+}$ is consistent with reports that $\mathrm{Pb}^{2+}$ entry into animal cells (Simons and Pocock, 1987; Tomsig and Suszkiw, 1991) as well as into plant cells (Huang and Cunningham, 1996) occurs, at least in part, through $\mathrm{Ca}^{2+}$-permeable channels.

We have taken a reverse genetic approach to studying the function of NtCBPs and AtCNGCs. We reported on the plasma membrane localization of NtCBP4, and on the phenotype of transgenic tobacco overexpressing this protein. We found that overexpression of NtCBP4 conferred tolerance to $\mathrm{Ni}^{2+}$ and hypersensitivity to $\mathrm{Pb}^{2+}$, associated with reduced $\mathrm{Ni}^{2+}$ uptake and enhanced $\mathrm{Pb}^{2+}$ uptake, respectively (Arazi et al., 1999). Because NtCBP4 is, to date, the only identified plant protein capable of modulating tolerance and accumulation of $\mathrm{Pb}^{2+}$, we investigated other possibilities of manipulating $\mathrm{Pb}^{2+}$ tolerance in tobacco by engineering NtCBP4.

In addition, we tested whether NtCBP4 homologues in Arabidopsis encode proteins that are potentially involved in $\mathrm{Pb}^{2+}$ transport. Comparison of $\mathrm{NtCBP4}$ with the $\mathrm{CNGC}$ gene family of Arabidopsis on a phylogenetic tree reveals that NtCBP4 is most related to the Arabidopsis CNGC1 gene. At the protein level, NtCBP4 is $82 \%$ similar and $75 \%$ identical to CNGC1. Therefore AtCNGC1 is a probable orthologue of NtCBP4 in Arabidopsis. Subsequently, we isolated a CNGC1 knockout mutant and investigated its response to $\mathrm{Pb}^{2+}$. Here we demonstrate that expression of a truncated NtCBP4 in the transgenic tobacco, and disruption of $C N G C 1$ in Arabidopsis resulted in improved tolerance to $\mathrm{Pb}^{2+}$.

\section{Results}

Transgenic tobacco plants expressing a truncated $\mathrm{NtCBP} 4$ exhibit improved tolerance to $\mathrm{Pb}^{2+}$

The C-terminal half of NtCBP4 contains a structurally conserved putative cyclic nucleotide-binding domain and a high-affinity calmodulin-binding site (Arazi etal., 2000). In contrast to the mammalian cyclic nucleotide-gated channels, where the calmodulin-binding domain is near the N-terminus of the protein (Varnum and Zagotta, 1997), in NtCBP4 the calmodulin-binding site coincides with the predicted $\alpha \mathrm{C}$-helix structure of the cyclic nucleotidebinding domain (Arazi etal., 2000). This helix is essential for cyclic nucleotide binding in related proteins from other organisms (Shabb and Corbin, 1992). We reasoned that removal of this important helix should disrupt the binding of both cyclic nucleotides and calmodulin, and could possibly render the channel inactive. Moreover, if plant NtCBP4-associated channels function as tetramers, as do mammalian cyclic nucleotide-gated channels (Liu etal., 1996), expression of an inactive subunit might have inhibitory effects on endogenous native NtCBP4-associated channel activities. As a first step to test this possibility, we prepared a truncated version of NtCBP4 from which a major part of the $\mathrm{C}$-terminal half, including the $\alpha \mathrm{C}$-helix, was removed (designated NtCBP4AC; Figure 1).

Transgenic plants expressing NtCBP4AC were prepared as described in Experimental procedures, selected on kanamycin, and transferred to the greenhouse for seed production. Seeds were plated on kanamycin-containing medium to select for progeny carrying the transgene. To investigate the possible effects of NtCBP4AC expression on
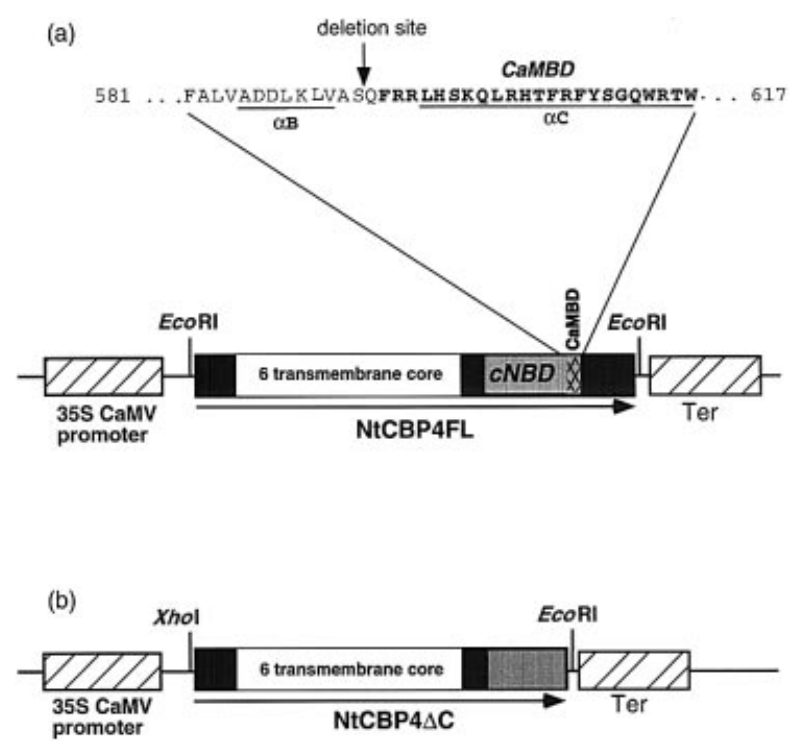

Figure 1. Schematic presentation of DNA constructs for expression of the full-length and C-terminal truncated NtCBP4 in transgenic tobacco.

(a) Description of the full-length NtCBP4 cDNA in a binary Ti plant transformation vector, as described by Arazi etal. (1999). Regions with the 35S CaMV promoter and the transcription termination sequence are shown in hatched boxes. The NtCBP4 six-transmembrane core, putative cyclic-nucleotide monophosphate-binding domain (cNBD, in grey; Arazi etal., 1999; Arazi etal., 2000) and the overlapping calmodulin-binding domain (CaMBD, cross-hatched; Arazi etal., 2000) are indicated. The amino-acid sequence of part of the cNBD is shown on top. The CaMBD (bold letters) and the $\alpha \mathrm{B}$ and $\alpha \mathrm{C}$ predicted helices (underlined) of the cNBD are according to Arazi etal. (2000). The downward arrow shows the site of the deletion used to create the $\mathrm{C}$-terminal truncated protein designated NtCBP4 $\Delta \mathrm{C}$ (amino acids $\mathrm{Met}_{1}-\mathrm{Ser}_{593}$ ). Numbers denote the terminal amino-acid residues shown in the sequence, based on Arazi et al. (1999) and Arazi et al. (2000).

(b) The NtCBP4 $\triangle \mathrm{C}$ cDNA in a binary Ti plant transformation vector. The $\mathrm{NtCBP} 4 \triangle \mathrm{C}$ cDNA, as described in (a), was prepared by cloning a corresponding PCR-amplified DNA fragment into the Xhol and EcoRI sites of the same vector used for the full-length NtCBP4. 
(a)

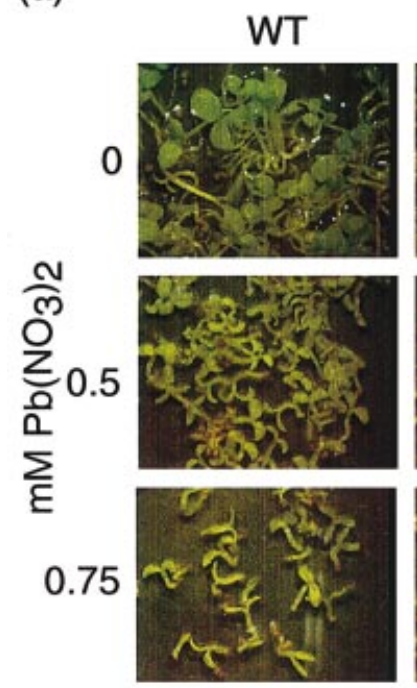

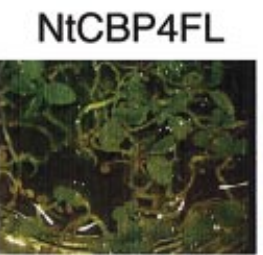
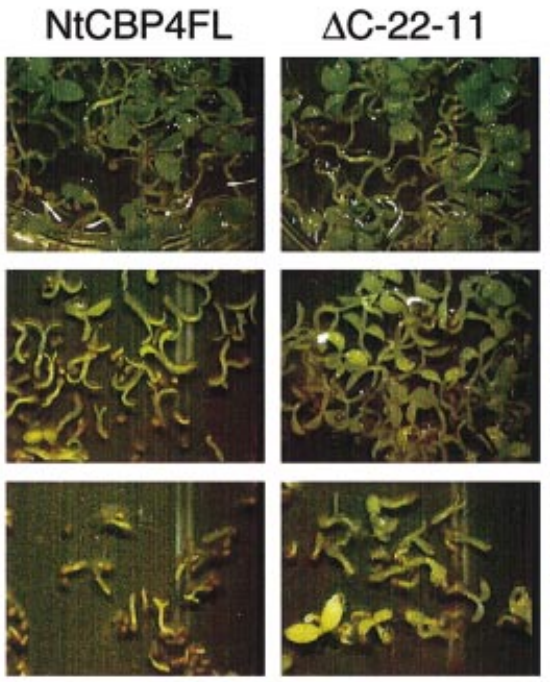

$\Delta \mathrm{C}-42-11$

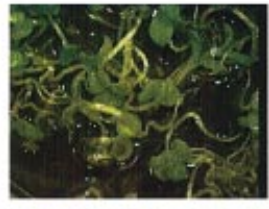

$\Delta \mathrm{C}-29$
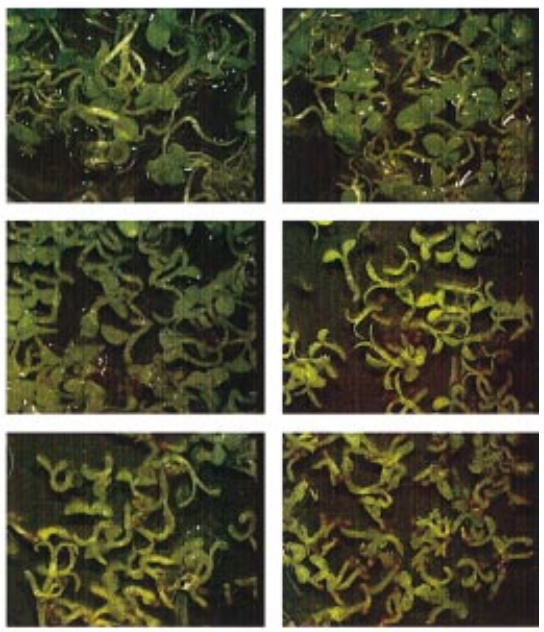

(b)
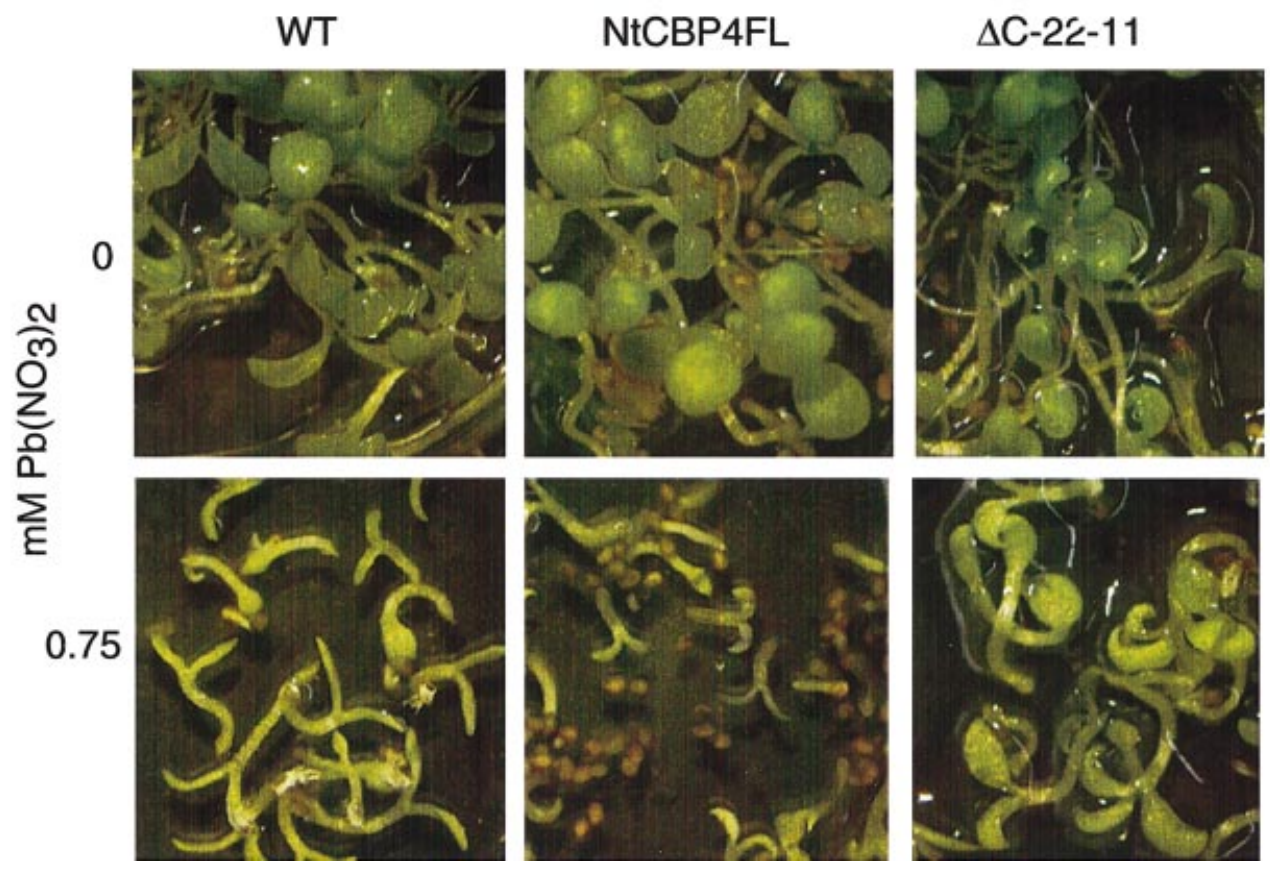

Figure 2. Transgenic tobacco seedlings expressing $\mathrm{NtCBP} 4 \Delta \mathrm{C}$ are tolerant to $\mathrm{Pb}^{2+}$.

(a) Seedlings of wild-type and transgenic tobacco expressing either the full-length NtCBP4 (NtCBP4FL; Arazi etal., 1999) or the NtCBP4 $\triangle \mathrm{C}$ mRNA (transgenic lines $\Delta \mathrm{C}-22-11, \Delta \mathrm{C}-42-11$ and $\Delta \mathrm{C}-29$ ) were germinated and grown in the presence of the indicated concentrations of $\mathrm{Pb}\left(\mathrm{NO}_{3}\right)_{2}$ in modified Blaydes solution ( $\mathrm{pH} 4.5$ ) for 12 days and then photographed.

(b) Photo enlargements of seedlings of wild-type and representative transgenic lines in the presence of 0 and $0.75 \mathrm{mM} \mathrm{Pb}\left(\mathrm{NO}_{3}\right)_{2}$.

plant response to $\mathrm{Pb}^{2+}, \mathrm{NtCBP} 4 \Delta \mathrm{C}$ transgenic lines were germinated in the presence of $\mathrm{Pb}\left(\mathrm{NO}_{3}\right)_{2}$ and their growth was compared with that of wild-type plants and of the transgenic line 49-79, which overexpresses the full-length NtCBP4 (designated NtCBP4FL) and is associated with $\mathrm{Pb}^{2+}$ hypersensitivity (Arazi etal., 1999). Eleven independent kanamycin-resistant lines (designated 42-11, 22-11, 29, 33-
$11,16-6,45-16,52-36,55-16,9-2,36-16,55-61)$ that express the NtCBP4 $\triangle C$ transgene were found to be more tolerant to $\mathrm{Pb}^{2+}$ than wild-type plants. Figure 2 shows the phenotype of three transgenic lines expressing the NtCBP4AC gene, and the wild-type and NtCBP4FL control lines, grown with or without $\mathrm{Pb}^{2+}$. The NtCBP4 $\Delta \mathrm{C}$ plants were more tolerant to the toxic metal than the wild-type seedlings, whereas 
the NtCBP4FL plants were more sensitive, as previously reported (Arazi etal., 1999). No differences were found between wild-type and tobacco transgenic lines in their response to other metals including $\mathrm{Na}^{+}, \mathrm{Zn}^{2+}, \mathrm{Mn}^{2+}, \mathrm{Cd}^{2+}$ and $\mathrm{La}^{3+}$.

The expression of NtCBP4FL and NtCBP4AC in transgenic plants is driven by the same promoter. However, we wished to confirm that their expression levels were similar, and to exclude the possibility that the expression of the NtCBP4AC mRNA abolished the expression of the endogenous native gene (for example by a gene silencing mechanism). We also wished to assess the correlation between NtCBP4AC expression and the phenotype. Northern hybridizations with total RNA samples were used for analysing steady-state mRNA levels (Figure 3a). We tested five independent kanamycin-resistant transgenic lines showing a $\mathrm{Pb}^{2+}$-tolerant phenotype, two kanamycin-resistant transgenic lines without an apparent phenotype, the transgenic line expressing the full-length NtCBP4 (Arazi etal., 1999), and the wild type. In the five

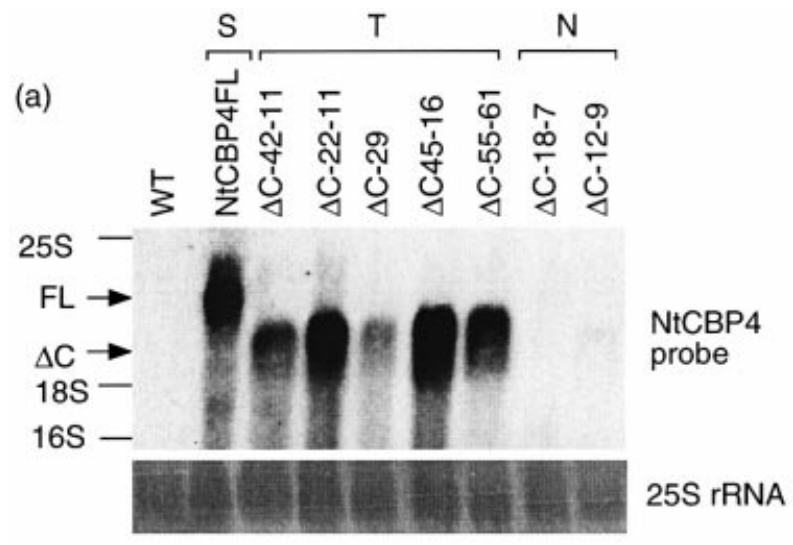

(b)

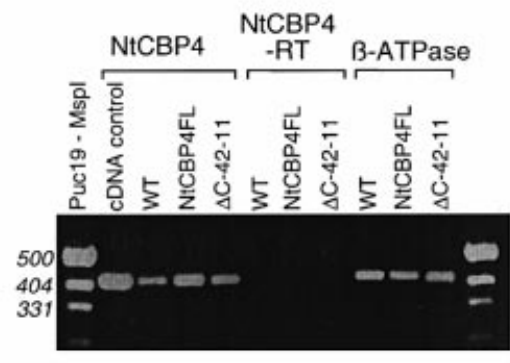

(c)

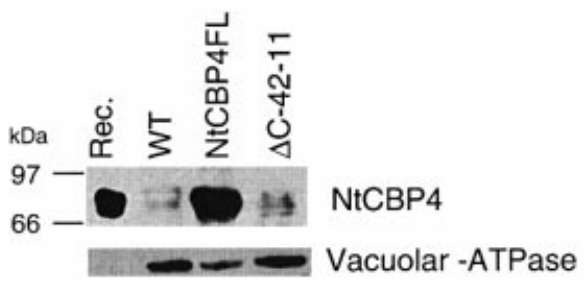

transgenic lines that showed a $\mathrm{Pb}^{2+}$ tolerance phenotype ( $\Delta \mathrm{C}-42-11, \Delta \mathrm{C}-22-11$ and $\Delta \mathrm{C}-29, \Delta \mathrm{C}-45-16$ and $\Delta \mathrm{C}-55-61$ ), the truncated-size mRNA was detected. It was easily distinguishable by its length (727 bases shorter) from the fulllength mRNA in line NtCBP4FL. Moreover, the expression levels of the full-length mRNA in NtCBP4, and the truncated mRNA in the NtCBP4 $\triangle \mathrm{C}$ lines were similar, with some variability in mRNA levels among the $\mathrm{NtCBP} 4 \Delta \mathrm{C}$ lines. Importantly, in the two kanamycin-resistant lines not showing a phenotype (lines $\Delta \mathrm{C}-18-7, \Delta \mathrm{C}-12-9$ ), expression of the truncated transgene was not detected. In addition, six independent transgenic tobacco lines expressing a non-relevant protein (glutamate decarboxylase) under the control of the $35 \mathrm{~S}$ CaMV promoter were indistinguishable from wild-type in response to $\mathrm{Pb}^{2+}$ (data not shown). These results indicate that the $\mathrm{Pb}^{2+}$ tolerance phenotype is associated with expression of the truncated $\mathrm{NtCBP} 4$ transgene. It should be noted that the endogenous native NtCBP4 mRNA in wild-type plants was not detected in the total RNA sample, due to its very low abundance (T. Arazi and $\mathrm{H}$. Fromm, unpublished results).

To ascertain that the expression of the endogenous native gene in $\mathrm{NtCBP} 4 \Delta \mathrm{C}$ transgenic plants was not silenced, we performed RT-PCR amplification with a set of primers that amplify a $404 \mathrm{bp}$ region of the full-length NtCBP4 mRNA, but not of the truncated mRNA. The RTPCR results (Figure $3 b$ ) show that a 404 bp DNA band was amplified from mRNA of the NtCBP $4 \Delta \mathrm{C}$ plants, indicating

Figure 3. Analysis of gene and protein expression in transgenic tobacco plants.

(a) Total RNA samples $(20 \mu \mathrm{g})$ from the indicated transgenic plants and wild type were separated by gel electrophoresis, blotted and hybridized with an NtCBP4-specific probe (upper panel). Arrows point to the fulllength NtCBP4 (FL) and the C-terminal truncated NtCBP4 $(\triangle C)$ mRNAs in the upper panel. The letters on top of the upper panel refer, for the transgenic lines indicated, to the hypersensitive (S), tolerant $(\mathrm{T})$ and normal $(\mathrm{N})$ phenotypes regarding response to $\mathrm{Pb}^{2+}$ relative to the wild type. The gel positions of the 25S, $18 \mathrm{~S}$ and $16 \mathrm{~S}$ ribosomal RNA bands are indicated. The $25 \mathrm{~S}$ ribosomal RNA band served as an internal standard (lower panel).

(b) Expression analysis of the full-length NtCBP4 mRNA in wild-type, $\mathrm{NtCBP} 4 \mathrm{FL}$ and NtCBP4 $\Delta \mathrm{C}$ (line $\Delta \mathrm{C}$-42-11) plants by RT-PCR. A $404 \mathrm{bp}$ DNA fragment corresponding to a region of the full-length NtCBP4 mRNA was amplified with the primers designated NtCBP4. Amplification of the corresponding region from the NtCBP4 cDNA clone served as a positive control (cDNA control). The amounts of polyA+ RNA were normalized using the expression of the tobacco gene encoding the $\beta$ subunit of mitochondrial $\mathrm{H}^{+}$-ATPase as a standard. Amplified DNA samples and DNA size markers (indicated in bp on the left) were fractionated by agarose gel electrophoresis, stained with ethidium bromide and photographed.

(c) Immunodetection of the full-length NtCBP4 in microsomes from wildtype, NtCBP4FL and NtCBP4 $\Delta \mathrm{C}$ (line $\Delta \mathrm{C}-42-11)$ plants. Microsomes $(20 \mu \mathrm{g}$ protein) and a sample of the full-length recombinant NtCBP4 expressed in insect cells (Rec; Arazi et al., 2000) were separated by SDS-PAGE and immunoblotted on a nitrocellulose membrane with polyclonal antiNtCBP4 antibodies (Arazi et al., 1999; Arazi et al., 2000). The positions of molecular weight markers are indicated. Immunodetection of vacuolar membrane $\mathrm{H}^{+}$-ATPase (Ward et al., 1992) served as an internal standard. 
(a)

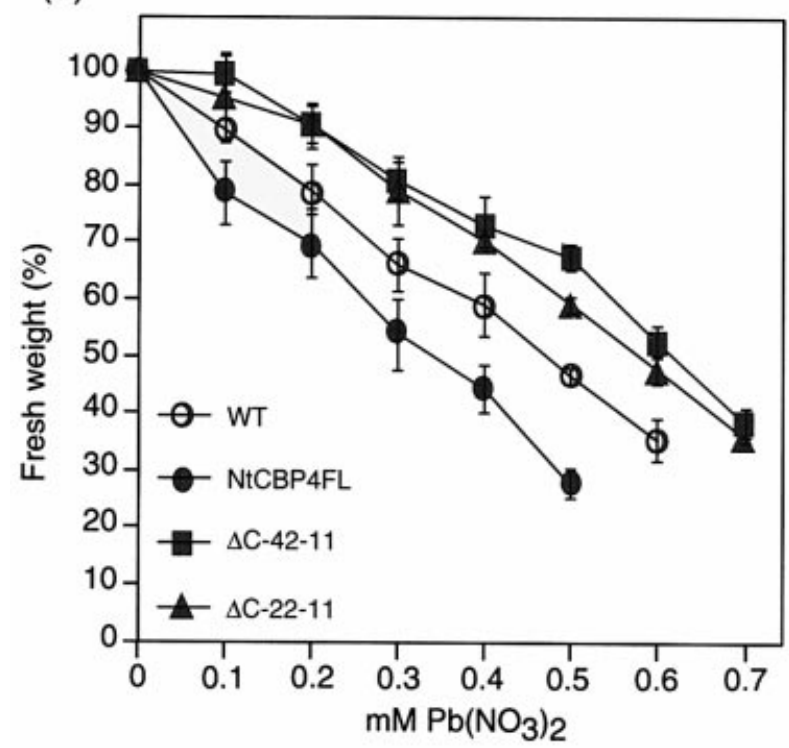

(b)

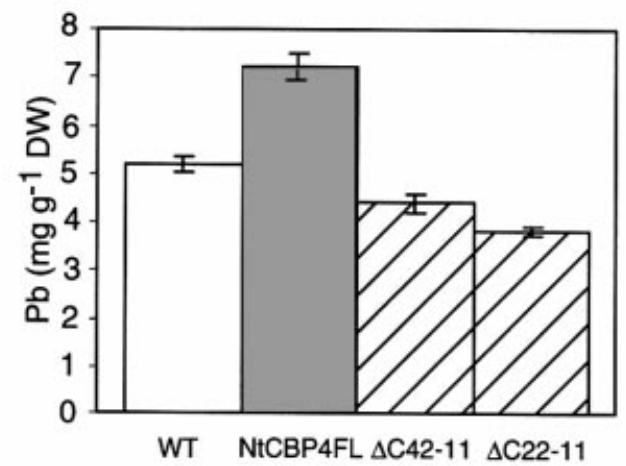

Figure 4. $\mathrm{Pb}^{2+}$ tolerance and accumulation in transgenic tobacco seedlings.

(a) Relative fresh weight of wild-type and transgenic tobacco seedlings expressing the full-length NtCBP4 mRNA (NtCBP4FL; Arazi etal., 1999), and two transgenic lines expressing the truncated NtCBP4 mRNA ( $\triangle \mathrm{C}-22$ 11 and $\Delta C-42-11)$ in the presence of different concentrations of $\mathrm{Pb}\left(\mathrm{NO}_{3}\right)_{2}$. Data are given as the mean $\mathrm{FW}$ of 150 seedlings for each concentration \pm SD of three independent experiments. Fresh weight of seedlings of each line grown without $\mathrm{Pb}\left(\mathrm{NO}_{3}\right)_{2}$ was set as $100 \%$.

(b) Lead accumulation in 12-day-old seedlings grown in the presence of $0.2 \mathrm{mM} \mathrm{Pb}\left(\mathrm{NO}_{3}\right)_{2}$. Seedlings were dried at $80^{\circ} \mathrm{C}$ for 3 days and lead content was determined by ICP-AES as described (Arazi et al., 1999). Data are the mean $\pm S D$ of three independent experiments.

that the endogenous native NtCBP4 mRNA was indeed present in these plants. This amplified band had the same mobility as the control band amplified from the NtCBP4 cDNA clone. Where reverse transcriptase was omitted (-RT), no amplification was detected, indicating that the observed amplifications described above originated from mRNA templates rather than from residual genomic DNA or from contaminating plasmid DNA in the samples. Amplification with primers corresponding to the nuclear gene encoding the $\beta$-subunit of mitochondrial $\mathrm{H}^{+}$-ATPase, as a control, indicated that similar amounts of poly $A^{+}$ mRNA were used in all reactions. Furthermore, immunoblot analysis using anti-NtCBP4 antibodies revealed that the endogenous native protein was expressed and present in membranes of NtCBP $4 \Delta \mathrm{C}$ lines, and the level of expression similar to that in the wild type. An example of protein analysis of line $\Delta \mathrm{C}-42-11$, the wild type and the NtCBP4FL line is shown in Figure 3(c). It should be noted that as the anti-NtCBP4 antibodies were raised against the C-terminal half of NtCBP4 (Arazi etal., 1999; Arazi etal., 2000), the truncated NtCBP4 protein could not be detected with these antibodies. Nevertheless, this analysis revealed that expression of NtCBP4AC does not result in silencing of the endogenous NtCBP4 gene. Rather, the apparent phenotype conferred by $N T C B P 4 \triangle C$ expression occurs in the presence of the full-length native endogenous NtCBP4.

Quantitative assessment of $\mathrm{Pb}^{2+}$ tolerance in NtCBP4AC transgenic seedlings, and $\mathrm{Pb}^{2+}$ accumulation

The extent of growth inhibition of seedlings by $\mathrm{Pb}^{2+}$ was evaluated by determining the fresh weight of seedlings grown in different concentrations of $\mathrm{Pb}\left(\mathrm{NO}_{3}\right)_{2}$ (Figure 4a). Whole-seedling fresh weight analysis revealed $50 \%$ inhibition at $0.34,0.47,0.58$ and $0.62 \mathrm{mM} \mathrm{Pb}^{2+}$ for NtCBP4FL, wild type, NtCBP4 $\Delta \mathrm{C}-22-11$ and NtCBP4 $\Delta \mathrm{C}-42-11$, respectively (Figure 4a). Moreover, statistical analysis revealed that the two NtCBP $4 \Delta \mathrm{C}$ lines were significantly more tolerant $(P<0.05)$ than the wild-type seedlings at $\mathrm{Pb}^{2+}$ concentrations of $\geqslant 0.1 \mathrm{mM}$. $\mathrm{Pb}^{2+}$ hypersensitivity of the $\mathrm{NtCBP} 4 \mathrm{FL}$ lines was also significantly different from the response of wild-type plants $(P<0.05)$, as previously described (Arazi etal., 1999).

One of the possible explanations for the $\mathrm{Pb}^{2+}$-tolerant phenotype of the NtCBP $4 \Delta$ C plants may be the attenuated uptake of the metal, as we have already demonstrated that $\mathrm{Pb}^{2+}$ hypersensitivity in NtCBP4FL plants was associated with enhanced accumulation of $\mathrm{Pb}^{2+}$ (Arazi et al., 1999). To test this possibility, we determined $\mathrm{Pb}^{2+}$ accumulation by ICP-AES in 12-day-old seedlings grown in the presence of $0.2 \mathrm{mM} \mathrm{Pb}\left(\mathrm{NO}_{3}\right)_{2}$. NtCBP4 $\Delta \mathrm{C}$ plants (lines $\Delta \mathrm{C}-42-11$ and $\Delta \mathrm{C}$ 22-11) showed a marked reduction in $\mathrm{Pb}^{2+}$ accumulation compared with wild-type seedlings $(P<0.05)$, whereas NtCBP4FL plants accumulated substantially more $\mathrm{Pb}$ $(P<0.05)$ than the wild type (Figure $4 \mathrm{~b})$, as previously described (Arazi et al., 1999).

The improved $\mathrm{Pb}^{2+}$ tolerance associated with attenuated $\mathrm{Pb}^{2+}$ accumulation in tobacco plants expressing a truncated NtCBP4 protein raised the possibility that the truncated protein inhibits the function of endogenous NtCBP4-associated channels. When the NtCBP4 amino- 
(a)

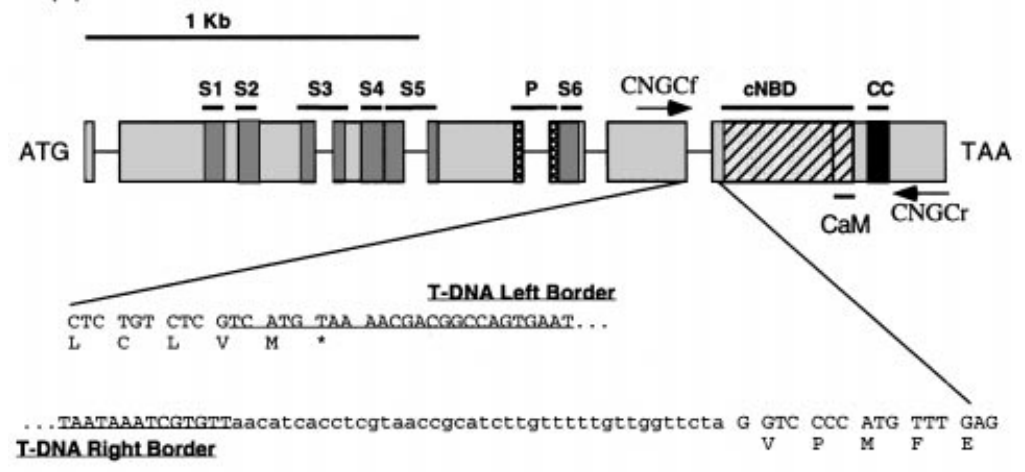

(b)

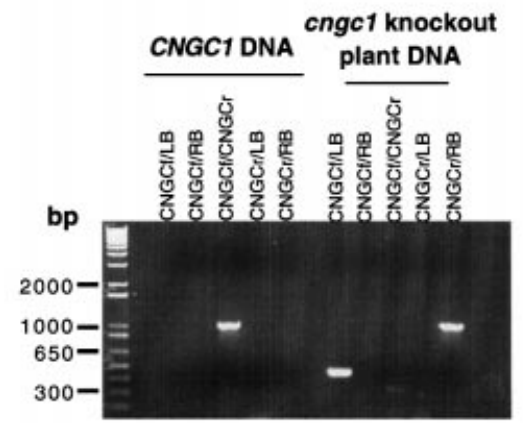

Figure 5. Genomic analysis of the T-DNA insertion in the Arabidopsis CNGC1 knockout line.

(a) CNGC1 gene map and T-DNA border sequences (T-DNA sequences are underlined). Exons are represented by boxes. S1 to S6, transmembrane hydrophobic domains; $\mathrm{P}$, predicted pore region; cNBD, cyclic nucleotide-binding site; CC, coiled coil helix; CaM, calmodulinbinding domain; CNGCf, CNGC1 forward oligonucleotide; $\mathrm{CNGCr}, \mathrm{CNGC1}$ reverse oligonucleotide.

(b) PCR analysis of cngc1 homozygous plants. Homozygous mutant and wild type (Arabidopsis thaliana ecotype Wassilewskija) DNA were subject to PCR amplification using CNGC1 gene-specific oligonucleotides (CNGCf and CNGCr) and TDNA left- and right-border oligonucleotides (LB and RB).

(c) Southern blot analysis of homozygous plants. DNA ( $2 \mu \mathrm{g}$ per lane) was digested by different restriction enzymes (the T-DNA contains one site for Pstl, EcoRl and HindIII and three sites for EcoRV, and blotted on nylon membrane. Hybridization was performed with a full-length radiolabelled TDNA probe. acid sequence is compared to the amino-acid sequences of the proteins encoded by the Arabidopsis CNGC genes, CNGC1 emerges as most similar to NtCBP4. We reasoned that if $A t C N G C 1$ is the orthologue of NtCBP4, then disruption of AtCNGC1 might confer $\mathrm{Pb}^{2+}$ tolerance in Arabidopsis. To assess this possibility we chose to isolate and analyse a T-DNA insertion mutant of the Arabidopsis CNGC1.

\section{Isolation of a CNGC1 knockout mutant and its response to $\mathrm{Pb}^{2+}$}

The full Arabidopsis CNGC1 coding sequence has recently been released and shown to be localized on chromosome 5 (BAC clone number MFH8, accession AB025622). The Versailles T-DNA library was screened by PCR for a knockout mutant using CNGC1-specific oligonucleotides, and T-DNA oligonucleotides localized in the left and right borders. One knockout line was isolated and shown to be disrupted in $\mathrm{CNGC1}$ by sequencing the T-DNA flanking regions (Figure $5 \mathrm{a}$ ). The T-DNA is inserted in front of the putative cyclic nucleotide-binding domain. The corresponding homozygous line was isolated and characterized by PCR (Figure $5 \mathrm{~b}$ ). Segregation analysis of the T-DNA insertion line revealed one insertion locus. DNA was extracted from homozygous plants, digested by several enzymes, and blotted on nylon membrane. Hybridization with a T-DNA probe revealed the presence of only one T-DNA insertion (Figure 5c). Moreover, DNA from homozygous plants was subjected to a PCR walking method (Devic etal., 1997) to clone any possible T-DNA border present in this line. Importantly, all cloned borders correspond to the CNGC1 insertion. No new T-DNA flanking regions were cloned from the knockout line, providing evidence for a single insertion site in the CNGC1 gene.

Subsequently, we analysed the response of seedlings of the homozygous cncg 1 mutant and wild type to different 
(a)

\section{$\mathrm{mM} \mathrm{Pb}\left(\mathrm{NO}_{3}\right)_{2}$}

0
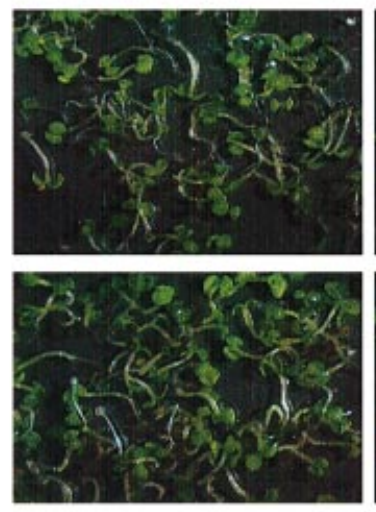

(b)

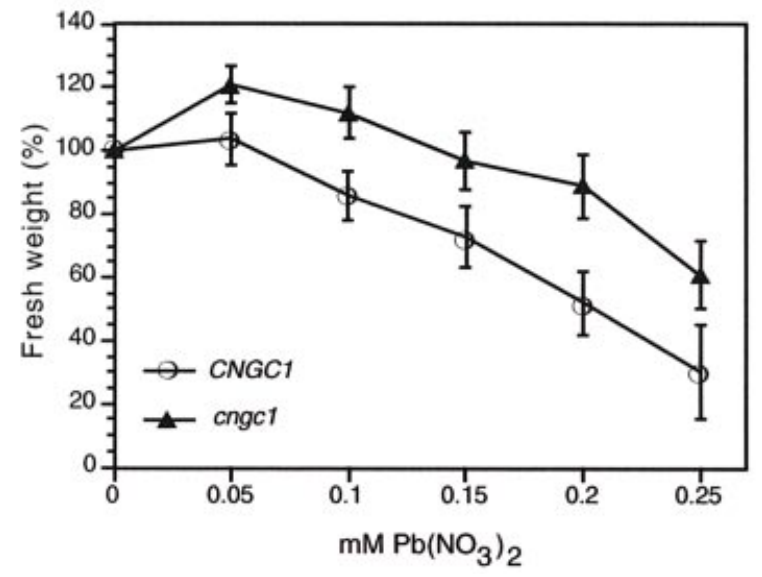

0.2
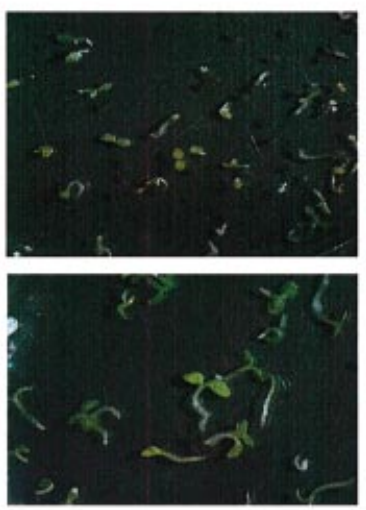

(c)

\subsection{5}

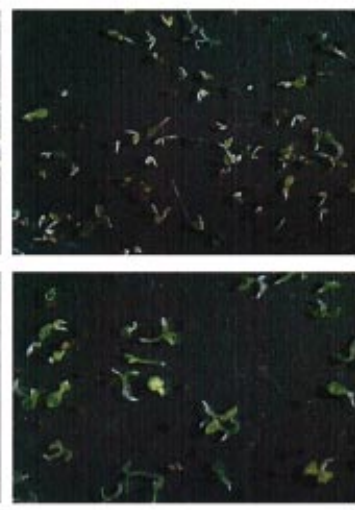

CNGC1

cngc1

Figure 6. Phenotypic analysis of the Arabidopsis cngc1 mutant in response to $\mathrm{Pb}^{2+}$

(a) Photographs of seedlings of wild-type and the $\mathrm{cngc1}$ mutant germinated and grown in the presence of the indicated concentrations of $\mathrm{Pb}\left(\mathrm{NO}_{3}\right)_{2}$ for 2 weeks.

(b) Relative fresh weight of wild-type and cngc1 mutant in the presence of different concentrations of $\mathrm{Pb}\left(\mathrm{NO}_{3}\right)_{2}$. Data are given as the mean $\mathrm{FW}$ of 150 seedlings for each concentration $\pm \mathrm{SD}$ of three independent experiments. Fresh weight of seedlings of each line grown without $\mathrm{Pb}\left(\mathrm{NO}_{3}\right)_{2}$ was set as $100 \%$. (c) Lead accumulation in 10-day-old seedlings grown in the presence of 0.05 and $0.15 \mathrm{mM} \mathrm{Pb}\left(\mathrm{NO}_{3}\right)_{2}$. Lead content was determined by ICP-AES as described in Experimental procedures. Data are the mean \pm SD of three independent experiments.

concentrations of $\mathrm{Pb}^{2+}$. Increasing $\mathrm{Pb}\left(\mathrm{NO}_{3}\right)_{2}$ concentrations adversely affected seedling development of both wild type and cngc1. However, in the presence of high concentrations of $\mathrm{Pb}^{2+}(0.2$ and $0.25 \mathrm{mM})$, the wild-type seedlings were chlorotic and their development was severely retarded compared with that of cngc 1 seedlings (Figure 6a). Similar results were obtained with two batches of wild-type seeds and three batches of mutant seeds from plants grown at different locations.

Quantitative analysis of $\mathrm{Pb}^{2+}$ tolerance of the cngc1 mutant is shown in Figure 6(b). This analysis revealed a statistically significant higher tolerance of the mutant line compared to the wild type. Importantly, analysis of the response of the Arabidopsis wild-type and mutant lines to $\mathrm{NiCl}_{2}$ revealed no apparent difference, suggesting that the cngc1 knockout specifically affects the response to $\mathrm{Pb}^{2+}$. However, this does not exclude possible specific effects on other ions, which have not been tested in this study. In addition we tested seedlings germinated and grown on agar plates. Seedlings were grown in the absence of $\mathrm{Pb}^{2+}$ for 7 days and then transferred to agar plates containing $\mathrm{Pb}^{2+}$. This growth regime also revealed a phenotype of 
$\mathrm{Pb}^{2+}$ tolerance of the cngc1 mutant in comparison to the wild type. Seedlings of the mutant showed less growth inhibition in the shoot, and retained more chlorophyll than the wild type at high $\mathrm{Pb}^{2+}$ concentrations (1 and $5 \mathrm{~mm}$ ). At these concentrations, the roots of both wild-type and cngc1 mutant ceased growing (data not shown). After 5 days at $5 \mathrm{mM} \mathrm{Pb}^{2+}$, seedlings of the cncg1 mutant retained twice as much chlorophyll as wild-type seedlings $(42.8 \pm 5.0$ and $20.6 \pm 1.6 \%$, respectively), relative to the non-stressed control seedlings.

Furthermore, we tested the $\mathrm{Pb}$ content in Arabidopsis seedlings exposed to $\mathrm{Pb}^{2+}$ for 2 weeks. At low concentrations of $\mathrm{Pb}^{2+}(0.05 \mathrm{mM})$ there was no significant difference between the cngc1 mutant and the wild type. At higher concentrations differences became apparent and significant (Figure 6c). At $0.15 \mathrm{mM} \mathrm{Pb}^{2+}$, the mean content of $\mathrm{Pb}$ in the wild type was $30 \%$ higher than in the mutant $(P<0.05)$. Thus, with respect to both $\mathrm{Pb}^{2+}$ tolerance and accumulation, the phenotype of the Arabidopsis cngc1 mutants is similar to that of transgenic tobacco plants expressing the truncated NtCBP4 protein.

\section{Discussion}

An emerging family of plant plasma membrane channel proteins resembling mammalian cyclic nucleotide-gated non-selective cation channels has been recently described in several plant species (Arazi etal., 1999; Köhler etal., 1999; Leng et al., 1999; Schuurink et al., 1998). We chose to use transgenic plants to study the function of the tobacco NtCBP4 protein, and T-DNA insertion mutagenesis to investigate the Arabidopsis homologue CNGC1. Our previous studies revealed that overexpression of NtCBP4 in tobacco plants conferred $\mathrm{Pb}^{2+}$ hypersensitivity and enhanced accumulation of $\mathrm{Pb}^{2+}$, suggesting the possible involvement of NtCBP4 in $\mathrm{Pb}^{2+}$ uptake across the plasma membrane (Arazi etal., 1999). Here we show that by expressing a modified version of NtCBP4 lacking presumed regulatory domains, the phenotype with respect to $\mathrm{Pb}^{2+}$ tolerance and accumulation is the opposite of that exhibited by plants overexpressing the full-length protein.

The mammalian cyclic nucleotide-gated channels function as tetrameric complexes (Liu et al., 1996). Therefore the inhibitory effect of $\mathrm{NtCBP} 4 \Delta \mathrm{C}$ on $\mathrm{Pb}^{2+}$ accumulation, and the concomitant improved tolerance to $\mathrm{Pb}^{2+}$, may have resulted from the formation of non-functional $\mathrm{NtCBP} 4 / \mathrm{NtCBP} 4 \Delta \mathrm{C}$ heteromeric complexes, as the endogenous native NtCBP4 gene was expressed in the background of the NtCBP4 $\triangle \mathrm{C}$ transgene (Figure 3c). However, as indicated, due to the fact that the anti-NtCBP4 antibodies were raised against the C-terminal half of NtCBP4 (Arazi et al., 1999; Arazi et al., 2000), the truncated NtCBP4 protein could not be detected with these antibodies. Further studies are required to assess the possible occurrence of full-length/truncated NtCBP4 heteromeric complexes in the NtCBP $4 \Delta \mathrm{C}$ transgenic plants. Nevertheless, we predicted that if the truncated protein causes the inactivation of endogenous NtCBP4-associated channels, then disruption of the Arabidopsis CNGC1 gene might cause a similar phenotype. Indeed, we isolated an Arabidopsis T-DNA insertion mutant of the CNGC1 gene. This mutant had characteristics similar to the tobacco plants expressing the truncated NtCBP4 with respect to the response to $\mathrm{Pb}^{2+}$. The phenotype of the cngc1 mutant was apparent when grown on different nutrient solutions, and irrespective of growth on liquid or solid substrate. Moreover, the phenotype was apparent when seeds were germinated in the presence of $\mathrm{Pb}^{2+}$, or when seedlings were exposed to $\mathrm{Pb}^{2+}$ only 1 week after germination. These results suggest the involvement of CNGC1 in $\mathrm{Pb}^{2+}$ transport across the plant plasma membrane, consistent with findings regarding NtCBP4 in tobacco.

It is important to note that the analysis described here was confined to the early stages of seedling development in tobacco and Arabidopsis. The response of older plants to $\mathrm{Pb}^{2+}$ and other metals requires further detailed analysis. In view of the fact that in Arabidopsis there are at least 19 CNGC-related genes (N. Bouché, unpublished results), the possible formation and complexity of CNGC heteromeric channels cannot be ignored. The composition of such heteromeric complexes may vary between cell types, during development, and in response to external physical and chemical stimuli. Heteromeric complexes might account for variations in regulation as well as in ion selectivity. In addition, CNGC proteins might differentially interact with distinct calmodulin isoforms (Köhler and Neuhaus, 2000), which could further affect their regulatory properties. Elucidating these issues requires a massive effort and a multidisciplinary research approach.

Because $\mathrm{Pb}^{2+}$ is a non-essential toxic metal to plants, the presumed NtCBP4- and CNGC1-associated ion transport systems are likely to have other, as yet unknown, physiological roles. On the basis of their primary structure, plant CNGCs are predicted to function as components of nonselective cation channels. The involvement of CNGC1 and CNGC2 in transport of potassium has already been reported (Köhler et al., 1999; Leng et al., 1999, respectively). Of particular interest to us is the possible involvement of $\mathrm{NtCBP} 4, \mathrm{CNGC} 1$ and other related plant proteins in $\mathrm{Ca}^{2+}$ signal transduction, either because of their expected regulation by $\mathrm{Ca}^{2+}$-calmodulin, and/or by being permeable to $\mathrm{Ca}^{2+}$, as in the case of mammalian cyclic nucleotidegated non-selective cation channels. A role in $\mathrm{Ca}^{2+}$ transport would be consistent with earlier reports identifying $\mathrm{Ca}^{2+}$-permeable channels as a pathway for $\mathrm{Pb}^{2+}$ entry into animal cells (Simons and Pocock, 1987; Tomsig and Suszkiw, 1991) and plants (Huang and Cunningham, 
1996), and with the recent demonstration of cyclic nucleotide-dependent $\mathrm{Ca}^{2+}$ entry into human embryonic kidney cells transfected with the Arabidopsis AtCNGC2 gene (Leng etal., 1999). We tested metal content in whole leaf extracts of wild-type and NtCBP4 $\triangle \mathrm{C}$ and NtCBP4FL transgenic tobacco grown in half-strength Hoagland's medium for 4 weeks (without heavy metal stress). There were no significant differences between the wild type and the transgenic lines in their content of a range of metals $(\mathrm{Ca}, \mathrm{K}$, $\mathrm{Mg}, \mathrm{Mn}, \mathrm{Zn}$ and $\mathrm{Fe}$ ). This may indicate that these CNGC channels have subtle effects on metal transport, which could be involved in signalling mechanisms.

Previously we reported that transgenic tobacco overexpressing the full-length NtCBP4 was tolerant to $\mathrm{Ni}^{2+}$ and hypersensitive to $\mathrm{Pb}^{2+}$, associated with reduced accumulation of $\mathrm{Ni}^{2+}$ and enhanced accumulation of $\mathrm{Pb}^{2+}$, respectively (Arazi etal., 1999). We hypothesized that the hypersensitivity to $\mathrm{Pb}^{2+}$ is the result of enhanced NtCBP4 channel activity. The opposite phenotype, resulting from the expression of a truncated NtCBP4 in tobacco and from disruption of CNGC1 in Arabidopsis, is consistent with this hypothesis. On the other hand, it would be difficult to associate the $\mathrm{Ni}^{2+}$-tolerance phenotype with channel activity. First, the Arabidopsis cngc1 mutant is not different from the wild type in response to $\mathrm{NiCl}_{2}$ (R. Sunkar and $\mathrm{H}$. Fromm, unpublished results). Secondly, we found that transgenic tobacco expressing the truncated NtCBP4 protein retains a certain degree of $\mathrm{Ni}^{2+}$ tolerance $(\mathrm{R}$. Sunkar and $\mathrm{H}$. Fromm, unpublished results). Therefore it is likely that the $\mathrm{Ni}^{2+}$ tolerance phenotype observed in transgenic tobacco (Arazi et al., 1999) is due to a property of the NtCBP4 protein, which is also present in the truncated protein, but is not directly associated with NtCBP4 channel activity.

In summary, we refined the analysis of the plasma membrane NtCBP4 tobacco protein in relation to $\mathrm{Pb}^{2+}$ tolerance. In addition, we found similar characteristics associated with the Arabidopsis CNGC1 gene. The transgenic tobacco plants and the Arabidopsis mutant described here provide unique research tools for addressing questions related to their physiological roles, as well as for elucidating mechanisms of heavy metal uptake and tolerance in plants.

\section{Experimental procedures}

\section{Preparation of transgenic plants}

Preparation of the DNA construct and of the transgenic tobacco plants for expression of the full-length NtCBP4 protein was recently described by Arazi etal. (1999). The DNA construct for the expression of the C-terminal truncated NtCBP4 (NtCBP4 $\Delta \mathrm{C}$ ) was prepared by cloning a PCR-amplified DNA fragment using the gene-specific oligonucleotides 5'-CCGCTGAGCTATGAATCACCGCCAAGACGAG-3' (sense) and 5'-GAAGGAATTCTTAAGAGGCTA-
CAAGCTTTAAATCAT-3' (antisense), spanning the coding region Met $_{1}-$ Ser $_{593}$ (Arazi etal., 1999), and containing Xhol and EcoRl restriction sites on the $5^{\prime}$ and $3^{\prime}$ termini, respectively. The XholEcoRI-digested DNA fragment was ligated into the corresponding sites of the binary vector described by Arazi et al. (1999). Transfer of the transgene to progeny was verified by germination on kanamycin, then by Northern hybridizations to confirm the expression of the NtCNBP4AC mRNA.

\section{Analysis of transgene expression by Northern hybridizations, RT-PCR and immunoblotting}

Total RNA was isolated from 3-week-old seedlings as described (Logemann et al., 1987). RNA fractionation, blotting and hybridization conditions were as described (Chen et al., 1994). A HindIII fragment from the cDNA of NtCBP4 (nucleotides 1113-2024, GenBank accession number AF079872) was labelled with $\left.{ }^{32} \mathrm{P}\right] \mathrm{dCTP}$ by random priming, and used as a probe. The same membrane was hybridized with a [ $\left.{ }^{32} \mathrm{P}\right] \mathrm{dCTP}$-labelled actin probe prepared by amplifying an actin gene fragment using the tomato actin gene (GenBank accession number U60480) specific primers 5'-TTGTGTTGGACTCTGGTGATGG-3' (sense) and 5'-AGCCAAGATAGAGCCTCCAATC-3' (antisense). Total RNA was passed through an oligo-dT column to enrich for polyA mRNA, and reverse transcriptase was used to prepare the corresponding cDNA templates. PCR amplification of a $404 \mathrm{bp} \mathrm{NtCBP4}$ fulllength-specific sequence was performed with the primers $5^{\prime}$ GACGACTTCACAGTAAGCAGC- $3^{\prime}$ and 5'-CACGACTAAAAATGCACTCAATC-3' (sense and antisense, respectively), which amplify a region spanning nucleotides 2044-2447 of the NtCBP4 CDNA (GenBank accession number AF079872). PCR amplification of the cDNA encoding the $\beta$-subunit of Nicotiana plumbaginifolia mitochondrial $\mathrm{H}^{+}$-ATPase (GenBank accession number X02868) served as a control, with the primers $5^{\prime}$-CTTACAGGTTTGACCGTGGCTGAGC-3' (sense), and 5'-TAGTGATCCTCTCCCAAAATGTGAGG-3' (antisense). Protein extraction, membrane fractionation and immunoblotting were performed as described (Arazi etal., 1999; Arazi etal., 2000).

\section{Plant culture, metal toxicity and metal accumulation assays}

Seeds of tobacco and Arabidopsis were sterilized by washing in $70 \%$ ethanol, then with chlorine, and subsequently rinsed with distilled water and, unless otherwise indicated, placed in Petri dishes containing modified Blaydes solution (Parrot and Bouton, 1990) $\mathrm{pH} 4.5$, with or without $\mathrm{Pb}\left(\mathrm{NO}_{3}\right)_{2}$. The seedlings were grown in a controlled growth room for 10 days (Arabidopsis) or $12-$ 13 days (tobacco), with a day/night cycle of $16 / 8 \mathrm{~h}$ at $25^{\circ} \mathrm{C}$. Seedlings were collected and their fresh weight measured, after which they were dried at $80^{\circ} \mathrm{C}$ for 3 days. Lead content was determined by ICP-AES as described (Arazi et al., 1999). Statistical analysis of the data was performed using ANOVA and the sum of squares simultaneous test procedure. For plants grown on agar plates, seeds were sterilized as described above and germinated on plates containing $25 \%(\mathrm{v} / \mathrm{v})$ Murashige and Skoog (MS) medium (Murashige and Skoog, 1962), 1\% (w/v) sucrose and $0.9 \%(w / v)$ agar (Sigma A-1296). Plants were grown for 7 days on vertically positioned plates with $16 / 8 \mathrm{~h}$ light/dark cycle with temperature of $25 / 20^{\circ} \mathrm{C}$, respectively, and light intensity of $40 \mu \mathrm{mol} \mathrm{m}{ }^{-2} \mathrm{sec}^{-1}$. Subsequently seedlings were transferred to plates containing $25 \%(\mathrm{v} / \mathrm{v}) \mathrm{MS}$ without $\mathrm{CaCl}_{2}$, with phosphate reduced to $0.01 \mathrm{mM}$ and with either 0,1 or $5 \mathrm{mM} \mathrm{Pb}\left(\mathrm{NO}_{3}\right)_{2}$. Shoots 
were harvested after 5 days. Chlorophyll was extracted with methanol and spectrophotometrically quantified. Total chlorophyll content ( $\mu \mathrm{g}$ chlorophyll $\mathrm{ml}^{-1}$ plant extract solution) was calculated as $22.5 \times A_{650}+4.0 \times A_{665}$.

\section{PCR screening strategy and knockout isolation}

We used the T-DNA inserted collection developed by G. Pelletier and co-workers (INRA, Versailles, France) made by infiltrating Arabidopsis thaliana ecotype Wassilewskija (Bechtold et al., 1993) with Agrobacterium tumefaciens strain C58 carrying the plasmid pGKB5 (Bouchez etal., 1993). This library is composed of 43584 individual lines, representing about 65000 independent insertions ( \pm 1.5 insertion loci per line). The DNA corresponding to $T_{2}$ or $T_{3}$ lines was extracted in pools of 48 lines (908 DNA pools). To isolate the cngc1 knockout, DNA pools were screened by PCR using gene-specific oligonucleotides (CNGCf: 5'-GAAGAGACGCAGAACAATGG-3'; CNGCr: 5'-CTGGCTCTGCTGGTTTCTGAAGTAGCATAG- $3^{\prime}$ ) and oligonucleotides anchored in the left and right borders of the T-DNA (RB oligonucleotide: $5^{\prime}$ CTGATACCAGACGTTGCCCGCATAA-3'; LB oligonucleotide: 5'CTACAAATTGCCTTTTCTTATCGAC- $3^{\prime}$ ). PCR reactions were run on an agarose gel, blotted onto nylon membranes which were hybridized with a T-DNA probe (RB plus LB) and a gene-specific probe (Arabidopsis EST 154M3T7 from Ohio Stock Center, corresponding to a partial CNGC1 cDNA clone). Positive PCR fragments hybridizing with both probes were isolated from agarose gel and sequenced. The 48 individual lines from the positive pool were then subjected to a new PCR and the corresponding knockout line was isolated.

\section{Acknowledgements}

This research was supported by a grant from the Israel Science Foundation to H.F., and by the French Embassy in Israel and the Académie d'Agriculture de France; R.S. and T.A. were recipients of fellowships from the Sir Charles Clore Foundation; and I.N.T. was the recipient of an AstraZeneca-funded studentship. We thank H. Sze for antibodies against vacuolar ATPase.

\section{References}

Arazi, T., Sunkar, R., Kaplan, B. and Fromm, H. (1999) A tobacco plasma membrane calmodulin-binding transporter confers $\mathrm{Ni}^{2+}$ tolerance and $\mathrm{Pb}^{2+}$ hypersensitivity in transgenic plants. Plant J. 20, 171-182.

Arazi, T., Kaplan, B. and Fromm, H. (2000) A high-affinity calmodulin-binding site in a tobacco plasma membrane channel protein coincides with a characteristic element of cyclic nucleotide-binding domains. Plant Mol. Biol. 42, 591-601.

Bechtold, N., Ellis, J. and Pelletier, G. (1993) In planta Agrobacterium mediated gene transfer by infiltration of adult Arabidopsis thaliana plants. Comptes R. Acad. Sci. Serie III Sci. la Vie, 316, 1194-1199.

Bouchez, D., Camilleri, C. and Caboche, M. (1993) A binary vector based on Basta resistance for in planta transformation of Arabidopsis thaliana. Comptes R. Acad. Sci. Serie III Sci. la Vie, 316, 1188-1193.
Chen, Y., Baum, G. and Fromm, H. (1994) The 58-kilodalton calmodulin-binding glutamate decarboxylase is a ubiquitous protein in petunia organs and its expression is developmentally regulated. Plant Physiol. 106, 1381-1387.

Devic, M., Albert, S., Delseny, M. and Roscoe, T.J. (1997) Efficient PCR walking on plant genomic DNA. Plant Physiol. Biochem. 35, 331-339.

Huang, J.W. and Cunningham, S.D. (1996) Lead phytoextraction: species variation in lead uptake and translocation. New Phytol. 134, 75-84.

Köhler, C. and Neuhaus, G. (2000) Characterisation of calmodulin binding to cyclic nucleotide-gated ion channels from Arabidopsis thaliana. FEBS Lett. 471, 133-136.

Köhler, C., Merkle, T. and Neuhaus, G. (1999) Characterization of a novel gene family of putative cyclic nucleotide- and calmodulin-regulated ion channels in Arabidopsis thaliana. Plant J. 18, 97-104.

Leng, Q., Mercier, R.W., Yao, W. and Berkowitz, G.A. (1999) Cloning and first functional characterization of a plant cyclic nucleotide-gated cation channel. Plant Physiol. 121, 753-761.

Liu, D.T., Tibbs, G.R. and Siegelbaum, S.A. (1996) Subunit stochiometry of cyclic nucleotide-gated channels and effects of subunit order on channel function. Neuron, 16, 983-990.

Logemann, J., Schell, J. and Willmitzer, L. (1987) Improved method for the isolation of RNA from plant tissues. Anal Biochem. 163, 16-20.

Murashige, T. and Skoog, F. (1962) A revised medium for rapid growth and bio assays with tobacco tissue culture. Physiol. Plant, 15, 473-497.

Ouyang, H. and Vogel, H.J. (1998) Metal ion binding to calmodulin: NMR and fluorescence studies. Biometals, 11, 213-222.

Parrot, W.A. and Bouton, J.H. (1990) Aluminium tolerance in alfalfa as expressed in tissue culture. Crop Sci. 30, 387-389.

Raskin, I. (1996) Plant genetic engineering may help with environmental cleanup. Proc. Natl Acad. Sci. USA, 93, 31643166.

Schuurink, R.C., Shartzer, S.F., Fath, A. and Jones, R.L. (1998) Characterization of a calmodulin-binding transporter from the plasma membrane of barley aleurone. Proc. Natl Acad. Sci. USA. 95, 1944-1949.

Simons, T.J.B. and Pocock, G. (1987) Lead enters bovine adrenal medullary cells through calcium channels. J. Neurochem. 48, 383-389.

Shabb, J.B. and Corbin, J.D. (1992) Cyclic nucleotide-binding domains in proteins having diverse functions. J. Biol. Chem. 267, 5723-5726.

Terry, N. and Banuelos, G. (1999). Phytoremediation of Contaminated Soil and Water. Boca Raton, FL: CRC Press.

Tomsig, J.L. and Suszkiw, J.B. (1991) Permeation of $\mathrm{Pb}^{2+}$ through calcium channels: fura-2 measurements of voltage- and dihydropyridine-sensitive $\mathrm{Pb}^{2+}$ entry in isolated bovine chromaffin cells. Biochim. Biophys. Acta, 1069, 197-200.

Varnum, M.D. and Zagotta, W.N. (1997) Interdomain interactions underlying activation of cyclic-nucleotide-gated channels. Science, 278, 110-113.

Ward, J., Reinders, A., Hsu, H. and Sze, H. (1992) Dissociation and reassembly of the vacuolar $\mathrm{H}^{+}$-ATPase complex from oat roots. Plant Physiol. 99, 161-169. 